\title{
EL DEBATE EN LA TEORÍA CONTEMPORÁNEA DE LA METÁFORA CONCEPTUAL: UNA EVALUACIÓN DEL ROL DEL CUERPO Y LA CULTURA MATERIAL
}

\section{THE DEBATE IN CONTEMPORARY CONCEPTUAL METAPHOR THEORY: AN EVALUATION OF THE ROLES OF THE BODY AND MATERIAL CULTURE}

\section{JORGE OSORIO*, CRISTIÁN SANTIBÁÑEZ ${ }^{* *}$}

Resumen: En este trabajo discutimos los cambios y alcances recientes que ha experimentado la teoría de la metáfora conceptual, preguntándonos tanto por las consecuencias internas en la teoría, como por los efectos de estos cambios para la disciplina lingüística. Especial atención le hemos dado a los cambios de énfasis que van desde entender la cognición metafórica como un proceso mental, hasta aquella perspectiva que centra su atención en el cuerpo y la cultura material. A partir de allí, hemos abordado con mayor detalle el concepto de esquema-imagen, así como el de affordance, para resaltar el rol que juegan el entorno y la cultura material en la práctica motora y lingüística, basada en una proyección metafórica bidireccional que va desde el cuerpo al ambiente, pero también de la cultura material hacia la cognición humana.

Palabras Clave: Teoría de la metáfora conceptual, affordance, esquema-imagen, lingüística, cultura material.

AвstRact: In this paper, we discuss the changes and recent developments in conceptual metaphor theory, reflecting on both the internal consequences for the theory as well as the effects of those developments on linguistics as a discipline. Special attention has been given to the changes of emphasis, which range from the understanding of metaphorical cognition as a mental process to a perspective that focuses on the body and material culture. In light of these developments, we address in greater detail the concepts of schema-image and affordance, in order to highlight the role played by the environment and material culture in motor and linguistic practices based on the bidirectional metaphorical projection from the body towards the environment, as well as from material culture towards human cognition.

KEYwords: Conceptual metaphor theory, affordance, image-scheme, linguistics, material culture.

Recibido: 21.04.2019. Aceptado: 20.01.2020.

* Doctor en Lingüística. Académico de la Universidad Católica de la Santísima Concepción, Concepción, Chile. Correo electrónico: josorio@ucsc.cl. Orcid: https://orcid.org/0000-0002-3592-4313.

** Doctor en Lingüística. Investigador del CIEDE, Universidad Católica de la Santísima de Concepción, Concepción, Chile. Correo electrónico: csantibanez@ucsc.cl. Orcid: https://orcid.org/0000-00016755-3468. 


\section{INTRODUCCIÓN}

T A BIBLIOGRAfía sobre metáfora, en la tradición occidental, cubre más de dos mil trescientos años, si consideramos como hito la Poética de Aristóteles. No corremos ningún riesgo al aseverar que, desde esa fecha hasta hoy, tal producción resulta inabarcable para cualquier investigador ${ }^{1}$. Esta profusión de estudios no es solo producto del tiempo transcurrido, sino sobre todo es una consecuencia del papel central que se le ha asignado a este fenómeno, tanto por la filosofía como por la lingüística, la psicología y otras ciencias de desarrollo más reciente (como las ciencias cognitivas o las neurociencias, por ejemplo).

El debate contemporáneo no puede soslayar la marcada influencia que ha tenido el denominado paradigma cognitivista en la redefinición del campo de estudios sobre la metáfora. A cuarenta años de la publicación de Metaphors we live by (Lakoff \& Johnson, 1980), ya resulta un lugar común valorar el impacto que ha tenido, en diversos campos, la teoría de la metáfora conceptual allí expuesta (en adelante, $\mathrm{TMC}^{2}$ ). La metáfora, en esta teoría, desempeña un papel crucial en el sistema cognitivo, asociado a la organización del conocimiento y a los procesos imaginativos que extienden y enriquecen la comprensión del mundo. Gibbs (1994) utiliza la expresión "ubicuidad de la metáfora" para señalar su innegable y, hasta cierto punto, abrumadora presencia en múltiples esferas. Más recientemente, este autor señala como principal respaldo para su afirmación el extenso cuerpo de hallazgos empíricos. Esta evidencia es resultado de la convergencia de disciplinas, enfoques y métodos diferentes que toman como objeto tanto el lenguaje (cotidiano y especializado) como el pensamiento abstracto y las experiencias emocionales y estéticas de las personas (Gibbs, 2008). En el último tiempo, esta aseveración ha sido respaldada por diversos estudios en el discurso (Semino, 2008; Pragglejaz Group, 2007; Cameron \& Deignan, 2006; Semino, Demjen, Hardie, Payne \& Rayson, 2018).

Dentro de los programas investigativos que han pretendido extender la teoría, quizá la propuesta más arriesgada provenga del propio Lakoff (2008), quien propuso introducir un giro hacia el modelamiento neuronal. Mientras la lingüística cognitiva asume el supuesto de que la estruc-

\footnotetext{
${ }^{1}$ Una búsqueda simple en la plataforma Google Académico (scholar.google.com), usando la clave "metaphor", nos arroja más de un millón de enlaces, solo en este siglo.

${ }^{2}$ Las denominaciones para la teoría son diversas (teoría contemporánea de la metáfora, teoría cognitiva de la metáfora, teoría conceptual de la metáfora). Preferimos "teoría de la metáfora conceptual", de amplio uso, porque expresa más claramente el objeto de estudio.
} 
tura semántica es un caso de estructura conceptual, afincando la noción de metáfora conceptual, la perspectiva neuronal pretende explicar cómo ocurren los apareamientos metafóricos a nivel neurológico y cómo esto se refleja en el procesamiento del lenguaje. Así, las descripciones lingüísticoconceptuales se confrontan con los modelos aportados por la neurobiología y la computación neuronal. El punto de partida es la presunción de que el sistema metafórico conceptual (conformado por un conjunto extenso de apareamientos metafóricos convencionales) existe físicamente en el cerebro y que una buena parte de los conceptos metafóricos está anclada en la experiencia corpórea (Lakoff, 2008).

En síntesis, la formulación inicial de la teoría conceptual, que considera a la metáfora como un "entramado de correspondencias", por ejemplo, entre el dominio GUERRA y el dominio DISCUSIÓN, debe replantearse en términos de un correlato neurológico, que requiere entender estas correspondencias como coactivaciones cerebrales. Es decir, la metáfora deja de ser una "construcción" mental y pasa a ser "algo que ocurre" en el cerebro. Se trata, entonces, de respaldar un principio más general, a nivel cognitivo, y no solo la dimensión lingüística. Así, las habilidades asociadas a los mecanismos de metaforización constituyen un eje relevante, no solo para la comprensión y producción del discurso, sino también para el desarrollo de la creatividad, el pensamiento inferencial y la resolución de problemas, entre otras habilidades.

El presente trabajo pretende contribuir al debate contemporáneo sobre el fenómeno metafórico, específicamente del estatus que la TMC le asigna y los desafíos teóricos que este estatus impone a los estudios del lenguaje, en el sentido de que éstos no solo deben centrarse en las expresiones (escritas, orales o comunicadas por otro medio) que los hablantes producen, sino también en el conjunto de condiciones materiales, psicológicas y sociales que las posibilitan.

En general, la evidencia multidisciplinar de que disponemos establece el desarrollo de una competencia (Littlemore, 2010) que, desde nuestra perspectiva, vincula estrechamente dos supuestos básicos: 1) El lenguaje se manifiesta como una proyección metafórica conceptual y 2) la proyección metafórica se manifiesta siguiendo una interacción de las personas que se vinculan materialmente entre sí y con el ambiente. A continuación desarrollamos ambos supuestos. El primer supuesto constituye el eje de la TMC, tal como fue expuesta por Lakoff \& Johnson (1980), y es el reconocimiento de la sistematicidad con que el fenómeno metafórico se evidencia en el lenguaje cotidiano. Sobre esta base, fue posible validar numerosos estudios descriptivos, centrados en la identificación de los dominios im- 
plicados en la relación metafórica, al tiempo que se conformaban extensos corpora de expresiones en diversas lenguas, motivando estudios comparados con impacto en diversas áreas de la lingüística descriptiva. No obstante, el denominado "compromiso cognitivo" (Lakoff, 1991) exigió dotar a todos estos hallazgos de un respaldo en el campo de las ciencias cognitivas, lo cual progresivamente tendió a relativizar los estudios de alcance descriptivo. Es decir, no resultaba suficiente la generalización de los llamados "mapeos metafóricos", pues el desafío era que tales procesos se explicaran como realidades mentales. De este modo, se pretendía dar cuenta de los hechos lingüísticos como hechos constitutivos de la cognición humana.

En la matriz programática de la lingüística cognitiva, esta pretensión implicaba redefinir el objeto de estudio, de modo tal que la TMC pasó a ser un espacio de convergencia teórica y empírica, una interdisciplina con toda propiedad. Esto remite a la segunda dimensión arriba indicada, esto es, la proyección metafórica se manifiesta siguiendo una interacción de las personas que se vinculan materialmente entre sí y con el ambiente.

Pese al auspicioso cuadro derivado de dichas extensiones, algunos lingüistas han sumado esfuerzos para mantener el fenómeno metafórico en el campo del lenguaje, asumiendo el anclaje conceptual como una fortaleza, y evitando de paso la excesiva dependencia de modelos tan externos como los de orden neurofisiológico (Rohrer, 2002). En este intento han potenciado el estudio de corpus y la integración de perspectivas sociocognitivas, que parecen restituir el componente fenomenológico de los actos de significación, dentro de los cuales se ubica la metáfora.

Así abierta la discusión, el presente trabajo tiene tres objetivos: 1) analizar el estado actual del debate en torno a la teoría conceptual de la metáfora que apunte a comprender el paso, primero, desde una perspectiva descriptiva inicial (la mente es el cuerpo), a otra con pretensiones explicativas externas a la descripción lingüística (el cuerpo en la mente), para luego pasar desde estas explicaciones externas a una visión completamente interactiva y materialista; 2) explicar por qué el estudio del fenómeno metafórico requiere un prisma evolutivo; y 3 ) reflexionar en torno a la necesidad que tiene el lingüista de trabajar multidisciplinariamente y ser competente en el desarrollo de estudios de carácter experimental.

Desde ya se debe indicar que parte importante de la óptica que orienta la presente discusión se desprende de la propuesta seminal de Metaphors we Live by (Lakoff \& Johnson, 1980), así como de la conceptualización expuesta en The Body in the Mind (Johnson, 1987) y en Philosophy in the Flesh (Lakoff \& Johnson, 1999), culminando con el reciente monográfico 
Embodied, Mind, Meaning and Reason (Johnson, 2017). Pese a que las tres últimas obras no corresponden, en específico, a estudios sobre metáfora, innegablemente constituyen el soporte para el despliegue de las extensiones de la TMC. De modo especial, y a partir de la última obra, es posible articular una visión sobre el fenómeno metafórico en la que podrían conciliarse los requerimientos más propios del estudio del lenguaje y los derivados del compromiso cognitivo original en la TMC. A riesgo de omitir consideraciones de importancia en la caracterización de la teoría, hemos preferido concentrarnos en algunos de los ejes que contribuyen a destacar el papel de la metáfora en la lingüística y en las ciencias en general. Para lograr los objetivos planteados, en el apartado 2 puntualizamos la doble valía del fenómeno metafórico en tanto objeto de estudio y en tanto método de interpretación; en el apartado 3 nos referimos a la dimensión más propiamente descriptiva del análisis metafórico conceptual, cuyo centro es el establecimiento de mapeos sistemáticos en múltiples dominios; en la sección 4 comentamos algunos aspectos de una fase más avanzada de la TMC, en la que se releva el anclaje corporal de los conceptos metafóricos y su incidencia en el procesamiento del lenguaje; en la sección 5 nos dedicamos a revisar críticamente el alcance de la noción esquema-imagen en tanto primera profundización del apartado 4; en la sección 6 reflexionamos en torno al problema que impone el conjunto de desafíos de asumir una dimensión material del funcionamiento cognitivo, lo que hacemos a través de la noción de affordance (Withagen, R. \& van Wermeskerken, 2010), y que corresponde a una segunda profundización del apartado 4; en la sección 7 , reflexionamos en torno a algunos elementos constitutivos de un ángulo evolutivo del fenómeno metafórico, para explicar la persistencia del funcionamiento metafórico en la cognición humana, retomando la idea de categorización cognitiva en tanto una estrategia evolutiva estable; y, por último, en las conclusiones entregamos una síntesis de nuestro acercamiento y aporte al debate actual en la teoría de la metáfora conceptual.

\section{LOS RECORRIDOS DE LA METÁFORA}

De Man (1978) sostiene que las metáforas, los tropos y el lenguaje figurado, en general, son un problema perenne y una molestia para el discurso filosófico, cuyo ejercicio muchas veces consistió en evitar el "daño epistemológico" que pudo propiciar la metáfora, en tanto que la metáfora es un artificio del lenguaje que obstaculiza un lenguaje más literal que se correspondería 
mejor con la búsqueda de la verdad. Si nos restringimos al ámbito semántico, este daño se produciría por la dificultad para fijar la referencia y, concomitantemente, para establecer el valor de verdad de los enunciados formados por expresiones metafóricas. En esta perspectiva (asociada normalmente al empirismo lógico), los términos del lenguaje refieren a los objetos y relaciones que se dan en el mundo, y sus enunciados representan, verdadera o falsamente, los estados de cosas en ese mundo (de Bustos, 2000).

Contra esta suerte de segregación epistemológica, con frecuencia el problema del significado metafórico obliga a defender definiciones teóricas, de menor o mayor impacto, pero ineludibles. Ricoeur (1978) observa que muchas teorías, aun cuando declaran concentrarse en el valor puramente informativo de las expresiones metafóricas, terminan por asignarle al sentimiento y la imaginación un valor semántico. Destacamos esta propuesta porque vincula una función perceptual (la captación de semejanzas) con una función lingüística (la de denominación), relación que ha motivado una interesante problematización en torno a la dimensiones emotivas, actitudinales y evaluativas del significado (Krzeszowski, 1990; Osorio, 2017), durante mucho tiempo marginadas de la semántica léxica (Lyons, 1997).

La imaginación, como cualidad del significado metafórico, explicaría su tratamiento diferenciado, ya no como un fenómeno marginal o como producto secundario, sino como eje para la comprensión del mundo. En efecto, es fácil apreciar la valoración del componente metafórico en el desarrollo del pensamiento científico, aunque a veces haya sido planteada en términos condicionales: "Toda ciencia debe partir de una metáfora y terminar en un álgebra”, señaló Black (1962, p. 242, trad. propia). Así, la metáfora no solo constituye un objeto de estudio ineludible para la semántica como ciencia de la construcción de significados, sino que también es posible verla como un principio que explicaría algunas de las formas más relevantes del conocer. En ambas facetas, la lingüística cognitiva ha señalado caminos muy productivos, potenciados por la consolidación de las ciencias cognitivas.

El desarrollo histórico de la TMC puede entenderse como un proceso de adscripción al compromiso cognitivo, durante el cual pueden observarse tendencias moderadas y otras más radicales. La TMC es una formulación metadisciplinaria, respecto de la que no cabrían restricciones de campo, tales como las implicadas en los estudios "puramente" lingüísticos. Esto es así porque el programa investigativo impulsado por la lingüística cognitiva asume que el lenguaje no es un sistema autónomo, sino que una pieza constitutiva del sistema cognitivo, por lo que no puede haber contradicción en las explicaciones que provengan del estudio de los diferentes componentes. 
La evidencia convergente constituye el principal criterio de validez de las generalizaciones científicas a las que aspira la lingüística cognitiva. El compromiso cognitivo implica una redefinición de estas generalizaciones, puesto que dependen de constructos tradicionalmente externos al lenguaje. Como comenta Ruiz de Mendoza (2004), se trata de una tesis más radical, respecto de otras alternativas de corte funcionalista, por ejemplo, pues requiere incorporar nociones y categorías provenientes de la psicología cognitiva, de la inteligencia artificial o de la neurobiología, como ya hemos adelantado. En el caso de la TMC, la búsqueda de evidencia convergente se centra en respaldar constructos centrales, tales como el de dominio conceptual o el de correspondencia metafórica.

\section{LA MENTE ES EL CUERPO}

Lakoff \& Johnson (1980) cambian el locus del problema desde el "sistema lingüístico" al "sistema cognitivo", lo que exige un marco explicativo de alcance mayor. Si bien el ensayo Metaphors we live by logra instalar la tesis de la existencia ubicua de la metáfora en la vida cotidiana, en la línea de la revalorización del lenguaje ordinario, la apuesta más fuerte de estos autores se concentra en el soporte experiencial de lo que llaman el "sistema metafórico conceptual". Lakoff (1993) afirma que este constructo tiene su respaldo empírico en tres fuentes principales: a) La evidencia de que los procesos metafóricos determinan la polisemia; b) la inferencia sistemática, y c) la evidencia psicolingüística disponible. En relación con la primera fuente empírica que informa el constructo propuesto por Lakoff (1993), Ibarretxe-Antuñano (2011) sostiene que la polisemia sistemática tiene su explicación en la motivación experiencial. De este modo, comienza a configurarse un marco de interpretación que supera la mera descripción de la mente en términos del cuerpo.

En efecto, uno de los principios a los que se adscribe la corriente cognitivista es el que señala que la estructura conceptual está corporeizada (Lakoff, 1991; Evans \& Green, 2006; Soriano 2012). Según este principio, la naturaleza de la organización conceptual surge de la experiencia corporal. Así, parte de lo que hace significativa la estructura conceptual es la experiencia con la que está asociada (Evans \& Green 2006, p. 157). Lakoff (1988) ya había postulado integrar en su noción de experiencia tanto las experiencias básicas sensorio-motrices y emocionales, como las sociales y otras que arrancan del hecho de ser humanos y vivir en una sociedad humana. La ex- 
periencia es, para este autor, un funcionamiento activo y una motivación de lo que es significativo en el pensamiento humano. Esta afirmación de base parece licenciar una explotación intensiva de los denominados "mapeos metafóricos" que, en su sentido estrictamente descriptivo, son orientados por la metáfora genérica LA MENTE ES EL CUERPO.

Siguiendo a Sweetser (1990), se puede indicar que el subsistema metafórico LA MENTE ES EL CUERPO da cuenta de un conjunto amplio de regularidades semánticas derivadas de la extensión unidireccional desde el dominio de lo físico hacia el dominio de lo mental. Los estudios llevados a cabo por Ibarretxe-Antuñano (2011) respecto de los verbos de percepción son una buena muestra de la estabilidad y sistematicidad de este macro apareamiento. LA MENTE ES EL CUERPO es una etiqueta, un descriptor no categorial que pretende expresar unitariamente esta sistematicidad de las correspondencias conceptuales.

Ejemplos de expresiones lingüísticas que dan cuenta, en el lenguaje cotidiano, de tales correspondencias, son los siguientes: (1) Claro que yo me huelo que la culpa de todo la tiene el sinvergüenza del marido; (2) en muchas ocasiones hemos tocado el tema de una posible intervención de las Fuerzas Armadas; (3) es una manera apresurada de ver las cosas. Como puede apreciarse, se trata de todo un sistema figurativo que se levanta sobre la experiencia sensorial, constituyéndose en la motivación para la emergencia de expresividad cotidiana. Consecuentemente, se puede señalar que LA MENTE ES EL CUERPO es una metáfora central en el sistema que comenzó a describirse hace cuarenta años, y ha permitido el desarrollo de un marco explicativo que podemos denominar proyectivo, de corte funcionalista. La garantía de este marco es que se sostiene en datos lingüísticos de alta cobertura y en el conocimiento de las propiedades del sistema perceptual y/o de la estructuración del espacio, por ejemplo. Es decir, establece explícitamente cuál es el anclaje de las metáforas en la experiencia corporal.

\section{EL CUERPO EN LA MENTE}

La noción de corporeización se ha planteado como la convergencia natural entre los hallazgos concretos de disciplinas complejas como la neurobiología y la reflexión teórica en filosofía de la mente (Varela, Thompson \& Rosch, 1991). A partir de su conocido ensayo The Body in the Mind: The Bodily Basis of Meaning, Imagination, and Reason, Johnson (1987) desarolla una línea crítica que invita a la superación del dualismo metafísico 
(cartesiano). Como resume Muñoz (2010): "No habría entonces un cuerpo y una mente separados e independientes, sino dos aspectos de un mismo proceso orgánico o corporal (programa motor, sensación, emoción, acción, pensamiento, lenguaje, significado, cultura)." (p. 105).

Visto de ese modo, para afirmar la noción de metáfora conceptual y hacerla compatible con la hipótesis de la corporeización, no basta con establecer subsistemas de apareamientos y definir cuáles son los dominios corporales, es decir, el anclaje de los conceptos. La exigencia, la más alta a efectos del cumplimiento del compromiso cognitivo, es determinar la naturaleza de ese anclaje y cómo actúa en la asignación de significado metafórico.

Minervino, Martín y Trench (2012) precisan que, según la tesis experiencialista del empleo de las metáforas conceptuales para la interpretación de los enunciados metafóricos, "el trabajo de comprensión de estas conlleva la realización de simulaciones sensorio-motoras de los conceptos base que se proyectan metafóricamente sobre los meta" (p. 22). En su discusión establecen la distinción entre esta tesis, que denominan fuerte, y otra (sostenida por ellos) que sería una versión débil. En la versión fuerte (formulada por la TMC) estas simulaciones son necesarias para la comprensión de las correspondientes expresiones metafóricas. En la versión débil estas simulaciones no serían necesarias, pero tendrían lugar en quienes están capacitados para realizarlas, enriqueciendo así el proceso de comprensión de las expresiones metafóricas.

Los elementos básicos de la simulación son entidades sensorio-motoras o "símbolos perceptivos" (Barsalou, 1999). Por ejemplo, la palabra "martillo" activa una simulación motora o manipulativa, pero quizá también visual, auditiva, táctil, etc. Más aún, también activaría disponibilidades (affordance) en el repertorio conductual del lector/oyente. Por ejemplo, las disponibilidades del martillo van desde la habitual de golpear clavos, a otras menos habituales, pero posibles en ciertas circunstancias como romper ladrillos, lanzarlo contra un cristal, o utilizarlo como arma. Las disponibilidades de "martillo", "clavo", "golpear", etc., se combinan en una simulación única, utilizando como indicios la estructura gramatical de la oración. Por ejemplo, en el enunciado "Pedro clava un clavo con el martillo", la construcción gramatical nos informa que el agente es Pedro, y el instrumento es "el martillo" y no "el clavo". Urrutia y de Vega (2012) nos han hecho ver que el significado se produce a partir de simulaciones perceptivas o motoras que cubren todo tipo de experiencia, y por ello es posible hablar de "cognición situada". De acuerdo con el énfasis de Urrutia y de Vega (2012), las teorías corpóreas han producido claras evidencias de la simulación motora en la 
comprensión de enunciados de dominios concretos, como el del ejemplo del martillo, pero no han llegado a conclusiones convergentes en el caso de conceptos abstractos, lo que introduce una incógnita respecto del lugar del fenómeno metafórico, desde el marco interpretativo provisto por la psicolingüística experimental. Para Valenzuela (2011), por otro lado, la TMC ha permitido avanzar en el conocimiento del significado corpóreo, aun cuando se mantienen abiertas algunas interrogantes, entre ellas, y de modo singular, la pregunta sobre el origen de las proyecciones entre estructuras. La complejidad inherente al fenómeno del significado entraña riesgos en la interpretación de los datos experimentales. Gibbs (2013) propone abandonar la noción "idealista" respecto de que los efectos experimentales obtenidos en estudios neurocientíficos deben ser necesariamente causados por mecanismos mentales específicos en la mente de las personas (la falacia "efectos=estructura"). Este hecho, coincidimos con el autor, reviste la mayor importancia, pues finalmente el fenómeno metafórico es siempre relativo a un individuo específico, un contexto específico, una lengua, etc.

\section{ESQUEMA-IMAGEN: COGNICIÓN MOTORA Y SISTEMA CONCEPTUAL}

Una de las grandes contribuciones de la TMC (Lakoff, 1987; Johnson, 1987) fue acuñar el término esquema-imagen para referirse a las proyecciones básicas que van desde el ejercicio motor y perceptivo de nuestros cuerpos al entendimiento conceptual y abstracto de ciertas interacciones y su etiquetado lingüístico. La idea esencial es que, dadas ciertas regularidades ambientales, los humanos experimentamos el entorno a partir de los patrones que nos permiten activar posibilidades con sentido. Así, las regularidades corporales (de percepción, movimiento, valoración) se combinan con patrones ambientales (arriba/abajo, dentro/fuera, adelante/atrás, iteración, balance, pérdida de balance, fuente-camino-meta, movimiento forzado, centro/periferia, derecho, curvo, etc.) que regulan nuestros recorridos e influencias sociales. Sin ir más lejos, el hecho de que operemos a partir de -y dentro de- campos gravitacionales genera experiencias recurrentes de arriba/abajo que provocan la noción de verticalidad (percibida y proyectada). Para dar otro ejemplo, del hecho de experimentar la simple y muy importante rutina del balance, y su pérdida, creamos una imagen de BALANCE que se proyecta sobre el balancear objetos y, metafóricamente, sobre estados internos del cuerpo, ecuaciones matemáticas, e incluso sobre 
nociones de justicia social y política. De la diaria rutina de experimentar con espacios y circuitos contenedores y objetos contenidos desarrollamos el esquema CONTENEDOR consistente en una frontera que define interior y exterior. Lo mismo ocurre con el esquema-imagen MOVIMIENTO. La característica especial de tales esquemas es que no están atados a una sola área sensorial y/o motora, sino que son multimodales, experimentados con distintas partes, y activados en distintas áreas cerebrales.

Se sostiene (Dodge \& Lakoff, 2005) que aun cuando se requieren más estudios de culturas comparadas, existirían ciertos candidatos de esquemasimagen que serían universales, como CONTENEDOR, FUENTE-CAMINOMETA, VERTICALIDAD, entre otros. El desafío para la lingüística tradicional es que tales esquemas son previos y subyacen al significado lingüístico de las expresiones, ya que son intrínsecamente estructuras corporeizadas significativas. El mensaje de los acercamientos cognitivistas al significado sería algo como: mira el ejercicio motor y perceptivo del cuerpo completo, y verás cómo comienza a funcionar el significado lingüístico.

El rendimiento semántico de los esquemas-imagen es de largo alcance y profundidad. Entre otras cosas, generan procedimientos inferenciales que, combinados, permiten marcar a través de proyecciones metafóricas (o metonímicas), procesos de otra índole. Por ejemplo, si analizamos el escenario básico que contiene el esquema-imagen FUENTE-CAMINO-META, veremos con facilidad (porque es de carácter muy mundano experimentar con ello) que los objetos viajan juntos cuando van a una misma velocidad orientados a una misma meta; que pueden distanciarse si uno de ellos es movido por una fuerza interviniente que lo saca de su camino, etc. A partir de esta dinámica percibida, que fija inferencias temporales y espaciales, el sistema conceptual que va adquiriendo tales orientaciones (estructurales), puede luego activar razonamientos que le permiten reorganizar experiencias de segundo nivel, esto es, de carácter más abstracto, como "íbamos juntos y para el mismo lado, pero se salió del camino de nuestra relación"”.

De modo que el desafío es patente: ¿De qué debe preocuparse el lingüista?, ¿de las expresiones lingüísticas cotidianas?, ¿o debe, al mismo tiempo, reconocer procesos cognitivos en la base? La respuesta es obvia a partir de lo que hemos venido mostrando y discutiendo. La lingüística no está inmune, disciplinariamente hablando, de incorporar nuevas teorías y métodos que le permitan compartir sus explicaciones con analistas de otras discipli-

\footnotetext{
${ }^{3}$ Expresión que se encuentra en el Corpus de Referencia del Español Actual (CREA), disponible en: http://www.rae.es/recursos/banco-de-datos/crea.
} 
nas. En relación con el análisis metafórico, requiere, por lo pronto, formar a sus futuros técnicos y profesionales en diseños experimentales que puedan ser replicados en otros lugares para lograr comparaciones culturales y ver así, por ejemplo, cuán robusta es una propuesta analítica en términos de su potencial predictor. Dicho de otra forma, se requiere formar y entrenar analistas en el manejo de diseños experimentales que, una vez validados contextual y culturalmente, permitan observar cuán extendido es el funcionamiento metafórico y cuáles son y dónde se encuentran las variaciones culturales específicas, tratando, respecto de estas últimas, de explicar sus peculiaridades.

\section{AFFORDANCE: INTERACCIÓN Y MATERIALIDAD}

La cultura material actual es más abundante que nunca en la historia de la humanidad, tanto física como simbólicamente (más artefactos, más emblemas). De modo que no se trata solo de entender que nuestra experiencia motora y perceptiva vaya incubando proyectiva e inferencialmente dinámicas de entendimiento entre el cuerpo y la mente, sino que además hemos sido capaces de dejar, extendida y distributivamente, en nuestra cultura material artefactos y expresiones cristalizadas que, entre otros beneficios, reducen el coste energético cognitivo de procesar información.

Como ya lo habíamos indicado en la sección 4, es con el concepto de affordance que la investigación especializada en psicología, filosofía y cognición se ha acercado a este hecho (Heras-Escribano, 2019; Smith, 2009; Withagen \& van Wermeskerken, 2010) ${ }^{4}$. El término puede ser descrito, primero, como una categoría analítica que refiere a la idea de que el entorno ofrece posibilidades de usos (beneficiosos o perjudiciales) a los humanos; en segundo lugar, y más específicamente, por affordance cabe entender la disponibilidad $y / u$ ofrecimiento cognitivo de uso que el ambiente y sus objetos tienen para el funcionamiento motor y perceptivo. Lo ejemplificábamos en la sección 4 con la idea de observar un martillo que ofrece usos diversos a partir de una disponibilidad percibida. La idea de affordance como disponibilidad/ofrecimiento ambiental se captura bien cuando entendemos que los objetos dan oportunidades de uso, en virtud de un diseño que invita y activa una determinada función en una ocasión particular.

\footnotetext{
${ }^{4}$ Fue acuñado por el psicólogo James Gibson en 1966, pero fue acotado y modificado por Donald Norman en 1988, quien introdujo la acepción que más se acerca al sentido que aquí usamos.
} 
Cuando miramos una taza que nos interesa alcanzar para su uso, en el viaje hacia ella para asirla, nuestro brazo y, particularmente, nuestra mano se va disponiendo de forma tal que nuestros movimientos se van limitando a la expresión del diseño.

Ahora bien, los objetos culturales (artefactos, pero también las ideas) que hemos creado a lo largo de la evolución han ido, precisamente, moldeando nuestras posibilidades cognitivas; vale decir, los hemos creado porque han dado resultados eficientes (una taza sirve mejor como ningún otro contenedor físico para tomar café, aunque pueda hacerlo en una botella plástica), pero luego ofrecen determinadas posibilidades de reacción al cuerpo y la mente, limitadas por su forma, color, olor y alcance. La materialidad cultural del entorno nos limita, pero al mismo tiempo descansamos en ella para reducir actos reflexivos constantes.

¿En qué aspectos esto se vincula con la construcción del significado y las teorías más contemporáneas de la metáfora? Se vincula de forma directa: nuestras proyecciones metafóricas motoras y perceptivas han ido edificando estructuras conceptuales que son propiedades colectivas que limitan, a la vez que facilitan, las interacciones comunicativas con sentido. Probablemente, las más importantes de las metáforas conceptuales de cada comunidad (materia siempre abierta a confirmación y/o falsación empírica) estén distribuidas y extendidas en el entorno. Candidatos predilectos de las metáforas conceptuales más importantes de una comunidad se manifiestan en los enunciados cristalizados (dichos, proverbios, máximas, etc.), que tienen un origen motor y perceptivo (como por ejemplo "más vale pájaro en la mano que cien volando") y que nos ofrecen, o se disponen, como constructos que limitan el uso para enmarcar (frame) de una forma un acontecer (entender de una determinada manera un contexto y situación particular), a la vez que reducen tiempo y energía cognitiva de carácter reflexivo para eliminar otras formas de marcar tal acontecer. Un ejemplo concreto de esto último fue el uso de la expresión "Arar con los bueyes que se tienen" enunciada por un político chileno, quien enfrentaba un conflicto político específico (véase el desarrollo pormenorizado del ejemplo en Santibáñez, 2010). Con esta expresión, el político hacía uso de un determinado frame (de conocimiento común y tradicional, proveniente de la materialidad observada en el trabajo con la tierra en el campo chileno) para generar mayor impacto y reducir el coste de interpretación en el destinatario (tanto político como ciudadano). A partir de la materialidad observada y experimentada con el trabajo de la tierra a través de animales que no son de fácil reemplazo, se generó una proyección metafórica transferida al lenguaje que se aplica allí 
donde se debe seguir realizando una labor costosa con los mismos protagonistas, porque son de difícil reemplazo. En el contexto del político, el reemplazo de los ministros del gobierno suponía una difícil negociación política.

Los enunciados cristalizados al estar disponibles en el ambiente, con la misma flexibilidad que tiene una taza (para servir café, sopa, matar una hormiga que pasa por debajo de mi brazo), moldean la posibilidad cognitiva de un individuo que encara un hecho abstracto y novedoso. El lenguaje, en tanto fenómeno material de varias capas (desde los fonemas, pasando por grafemas y distribuidos en el ambiente), se va proyectando al nivel conceptual, el que, a su vez, nos permite apreciar cuán dependientes somos de lo comunitario y cuán físicos somos fenoménicamente.

\section{LA PERSISTENCIA DEL FUNCIONAMIENTO METAFÓRICO EN LA COGNICIÓN HUMANA: ELEMENTOS EVOLUTIVOS}

En los desarrollos previos, hemos apuntado a la ubicuidad de la metáfora en tanto rasgo saliente de parte importante de la práctica cognitiva humana. Se trata de una ubicuidad motora, perceptiva, lingüística y simbólica en general. Su raigambre evolutiva, no obstante, es más primitiva de lo que se pudiera pensar a primera vista.

La evidencia arqueológica (Coward \& Gamble, 2010; Malafouris, 2013) demuestra que fue en la era neolítica cuando se enriqueció la cultura material, vale decir, fue en esa época cuando hubo una potenciación de las capacidades cognitivas. Una de las claves del cambio fue el proceso de metaforización en la cultura material en vez del proceso semiótico atribuido a la naturaleza de la cultura. Desde la perspectiva arqueológica, valga recordar, el objeto precede a la palabra. De modo que, incluso desde el punto de vista arqueológico, la experiencia homínida material temprana obedeció a un patrón metafórico de entender una cosa por otra. Prueba de ello es que el ensamble en la fauna fue percibido y transportado metafóricamente al entendimiento de las relaciones con otros agentes; el uso de los huesos de personas muertas se utilizó como mapas mentales respecto de lugares con cierto significado y relevancia, formando así una evidente comprensión metonímica a partir de una materialidad orientada hacia, o por, el espíritu.

Ahora bien, si la función de toda cognición es la predicción al servicio de la acción (Barrett, 2015), entonces, el fenómeno metafórico es una casi perfecta manera de coordinación que permite planear lo que viene, ya que 
funciona como un repositorio conceptual y lingüístico de las interacciones entre humanos y el entorno que han servido para enmarcar y resolver. Dicho de otra forma, el proceso de metaforización, que tiene como uno de sus ejes la proyección metafórica de la cultura material al lenguaje y el mundo simbólico, se convierte en una estrategia evolutiva estable que se inserta en los procesos cognitivos.

¿Cuáles son aquellos procesos cognitivos que manifiestan la estrategia evolutiva estable asociada al proceso de metaforización? Como la literatura clásica sobre el fenómeno cognitivo demostró hace varias décadas (Rosch, 1975, 1978), uno de ellos es la capacidad de categorizar, que precisamente apoya el ordenamiento del presente para planear el futuro. Cabe recordar que categorizar es un mecanismo cognitivo que ordena entidades semánticas u objetuales de acuerdo con atributos salientes o prototípicos que jerarquizan información. Este mecanismo cognitivo se estructura a partir de dos sub-principios: economía cognitiva y estructuración del mundo percibido. Una vez dentro del funcionamiento categorizador, un sub-mecanismo es el ordenamiento por inclusividad, esto es, la fuerza de una categoría por englobar, atraer, depositar en sí, una mayor cantidad de atributos comunes en el marco de una familia de conceptos que refieren a un mismo evento simbólico o material, y que ayuda a orientar al agente que lo utiliza en su efecto motor y comunicativo.

En el marco del debate más actual acerca de la teoría de la metáfora conceptual, parece haberse instalado una dicotomía innecesaria: mantenerse en el estudio de las expresiones lingüísticas, como única dimensión material, o estudiar los conceptos y su materialidad neurobiológica. Nos parece que una mirada sobre los aspectos evolutivos del lenguaje y la cognición puede permitir desatar este y otros nudos en la investigación lingüística cognitiva. En cuanto a la metáfora, seguramente se mantendrá en un lugar de privilegio para dilucidar cómo ocurre la integración mente/cuerpo.

\section{CONCLUSIONES}

La versión estándar de la TMC, así como su último desarrollo vinculado a la dimensión neurocientífica, e incluso la aproximación que aboga por un ángulo estrictamente lingüístico al fenómeno metafórico (Kövecses, 2008), tienen respaldos empíricos, experimentales y conceptuales de años de trabajo, fundamentalmente, cuando dialogan con disciplinas como la psicología, las ciencias cognitivas y la filosofía (prueba de ello es, además, 
Metaphors we live by, ensayo escrito por un lingüista y un filósofo). En este contexto, ¿en qué queda el desafío y la tarea de los/as lingüistas? Como hemos querido implicar, queda en la promisoria posición de perfeccionarse en el trabajo multidisciplinar, en la incorporación curricular de contenidos relativos a cómo crear y gestionar diseños experimentales; queda en la imaginativa tarea de abordar el funcionamiento cognitivo y lingüístico de grupos humanos como los infantes, para estudiar, por ejemplo, cómo la competencia lingüística se va configurando tempranamente en un delicado balance entre operación motora y perceptiva y comunicación simbólica. Dicho en otras palabras, nuestra sugerencia es que el trabajo de los lingüistas debe también preocuparse por el estudio del desarrollo del uso de la metáfora, ontogénicamente hablando, para ir aportando a una visión más completa del funcionamiento de lo humano, filogénicamente hablando.

Desde el punto de vista analítico, nuestro énfasis radica en sugerir que el trabajo lingüístico debe transitar, en la explicación de la construcción de significado a través del funcionamiento metafórico, desde la mente en el cuerpo, pasando por el cuerpo en la mente, para alcanzar el problema de cómo la disponibilidad ambiental, tanto física como simbólica, está en ambos: en el cuerpo y en la mente. Por este motivo es que hemos sugerido el concepto de affordance (disponibilidad/ofrecimiento), a través del que quizá pueda encontrarse una explicación más robusta de la manera en que la cultura material activa a la vez que delimita las elecciones de esquemaimagen y expresiones metafóricas en hablantes naturales de una lengua. Ciertamente, para todo aquello se requiere colaborar sin barreras disciplinarias.

\section{REFERENCIAS}

Barrett, C. (2015). The Shape of Thought. How Mental Adaptations Evolve. New York, United States: Oxford University Press.

Barsalou, L. (1999). Perceptual Symbol Systems. Behavioral and Brain Sciences 22, $577-660$.

Black, M. (1962). Models and metaphors. Ithaca, New York, United States: Cornell University Press.

Cameron, L. \& Deignan, A. (2006). The Emergence of Metaphor in Discourse. Applied Linguistics 27(4), 671-690.

Coward, F. \& Gamble, C. (2010). Metaphor and Materiality in Early Prehistory. En Malafouris, L. y Renfrew, C. (eds.). The Cognitive Life of Things (pp. 4758). Cambridge, United Kingdom: McDonald Institute for Archaeological Research. 
De Bustos, E. (2000). La metáfora: Ensayos transdisciplinares. Madrid, España: Fondo de Cultura Económica.

De Man, P. (1978). The Epistemology of Metaphor. Critical Inquiry 5(1), 13-30.

Dodge, E. \& Lakoff, G. (2005). Image Schemas: From Linguistic Analysis to Neural Grounding. En Hampe, B. y Grady, J. (eds.). From Perception to Meaning: Image Schemas in Cognitive Linguistics (pp. 57-92). Berlin, Germany: Mouton de Gruyter.

Evans, V. \& Green, M. (2006). Cognitive Linguistics: An Introduction. Edinburgh, United Kingdom: Edinburgh University Press.

Gibbs, R. (1994). Poetics of Mind: Figurative Thought, Language, and Understanding. Cambridge: Cambridge University Press.

Gibbs, R. (2008). Metaphor and Thought. State of Art. The Cambridge Handbook of Metaphor and Thought (pp. 3-13). Cambridge, United Kingdom: Cambridge University Press.

Gibbs, R. (2013). Do Conceptual Metaphors Emerge from Metaphoric Language? Journal of Cognitive Science 14, 319-344.

Heras-Escribano, M. (2019). The Philosophy of Affordances. London, United Kingdom: Palgrave Macmillan.

Ibarretxe-Antuñano, I. (2011). Metáforas de la percepción: Una aproximación desde la lingüística cognitiva. En Santibáñez, C. y Osorio, J. (eds.). Recorridos de la metáfora: mente, espacio y diálogo (pp. 141-166). Concepción, Chile: Cosmigonon.

Johnson, M. (1987). The Body in the Mind: The Bodily Basis of Meaning, Imagination, and Reason. Chicago, Illinois, United States: University of Chicago Press.

Johnson, M. (2017). Embodied, Mind, Meaning and Reason. Chicago, Illinois, United States: University of Chicago Press.

Kövecses, Z. (2008). Conceptual Metaphor Theory: Some Criticisms and Alternative Proposals. Annual Review of Cognitive Linguistics 6, 168-184.

Krzeszowski, T. (1990). The Axiological Aspect of Idealized Cognitive Models. En Tomaszczyk, J. y Lewandowska-Tomaszczyk, B. (eds.). Meaning and Lexicography (pp. 135-165). Amsterdam, Netherlands: John Benjamins.

Lakoff, G. (1987). Women, Fire and Dangerous Things: What Categories Reveal about the Mind. Chicago, Illinois, United States: University of Chicago Press.

Lakoff, G. (1988). Cognitive semantics. En Eco, U. (ed.), Meaning and Mental Representations (pp. 119-154). Bloomington, Indiana, United States: Indiana University Press.

Lakoff, G. (1991). Cognitive versus Generative Linguistics: How Commitments Influence Results. Language \& Communication 11(1-2), 53-62.

Lakoff, G. (1993). The Contemporary Theory of Metaphor. En Ortony, A. (ed.) Metaphor and Thought (2a. edic., pp. 202-251). New York, United States: Cambridge University Press.

Lakoff, G. (2008). The Neural Theory of Metaphor. En Gibbs, R. (ed.), The 
Cambridge Handbook of Metaphor and Thought (pp. 17-38). Cambridge, United Kingdom: Cambridge University Press.

Lakoff, G. \& Johnson, M. (1980). Metaphors we Live by. Chicago, Illinois, United States: The University of Chicago Press.

Lakoff, G. \& Johnson, M. (1999). Philosophy in the Flesh: The Embodied Mind and its Challenge to Western Thought. New York, United States: Basic Books.

Littlemore, J. (2010). Metaphoric Competence in the First and Second language: Similarities and Differences. En Putz, M. \& Sicola, L. (eds.), Cognitive Processing in Second Language Acquisition (pp. 293-316). Amsterdam, Netherlands/Philadelphia, United States: John Benjamins.

Lyons, J. (1997). Semántica lingüística. Una introducción. Barcelona, España: Paidós.

Malafouris, L. (2013). How Things Shape The Mind. A Theory of Material Engagement. Cambridge, Massachusetts, United States: The MIT Press.

Minervino, R., Martín, A. y Trench, M. (2012). La comprensión de metáforas no requiere realizar simulaciones sensorio-motoras del dominio base. $R e$ vista Latinoamericana de Psicología 44(3), 23-34.

Muñoz, C. (2010). El cuerpo en la mente. La hipótesis de la corporeización del significado y el dualismo. Praxis 18, 91-106.

Osorio, J. (2017). Dimensión axiológica del significado y discurso argumentativo: un análisis exploratorio. Cogency 9(2), 47-65.

Pragglejaz Group. (2007). MIP: A Method for Identifying Metaphorically Used Words in Discourse. Metaphor and Symbol 22(1), 1-39.

Ricoeur, P. (1978). The Metaphorical Process as Cognition, Imagination, and Feeling. Critical Inquiry 5(1), 143-159.

Rohrer, T. (2002). The Cognitive Science of Metaphor from Philosophy to Neuroscience. Theoria et Historia Scientiarum VI(1), 27-42.

Rosch, E. (1975). Cognitive Representations of Semantic Categories. Journal of Experimental Psychology 104, 192-233.

Rosch, E. (1978). Principles of Categorization. En Rosch. E. y Lloyd, B. (eds.). Cognition and Categorization (pp. 27-48). Hillsdale, New Jersey, United States: Erlbaum.

Ruiz de Mendoza, F. (2004). Principios cognitivos y pragmáticos del procesamiento y la comprensión. Arbor CLXVII(697), 1-28.

Santibáñez, C. (2010). Metaphors and Argumentation: The Case of Chilean Parliamentarian Media Participation. Journal of Pragmatics 42(4), 973-989.

Semino, E. (2008). Metaphor in Discourse. Cambridge, United Kingdom: Cambridge University Press.

Semino, E., Demjen, Z., Hardie, A., Payne, S. A., \& Rayson, P. E. (2018). Metaphor, Cancer and the End of Life: A Corpus-based Study. London, United Kingdom: Routledge.

Smith, B. (2009). Toward a Realistic Science of Environments. Ecological Psychology 21(2), 121-130.

Soriano, C. (2012). La metáfora conceptual. En Ibarretxe-Antuñano, I. y Va- 
lenzuela, J. (eds.), Lingüística cognitiva (pp. 97-121). Barcelona, España: Anthropos.

Sweetser, E. (1990). From Etymology to Pragmatics: Metaphorical and Cultural Aspects of Semantic Structure. Cambridge, United Kingdom: Cambridge University Press.

Urrutia, M. y De Vega, M. (2012). Lenguaje y Acción. Una revisión actual a las teorías corpóreas. Revista de Lingüística Teórica y Aplicada (RLA) 50(1), 39-67.

Valenzuela, J. (2011). Sobre la interacción lengua-mente-cerebro: la metáfora como simulación corporeizada. Revista de Investigación Lingüística 14, 109126.

Varela, F., Thompson, E. \& Rosch, E. (1991). The Embodied Mind: Cognition Science and Human Experience. Cambridge, Massachusetts, United Kingdom: MIT Press.

Withagen, R. \& van Wermeskerken, M. (2010). The Role of Affordances in the Evolutionary Process Considered: A Niche Construction Perspective. Theory and Psychology 20(4), 489-510. 